\title{
Prediction of novel long non-coding RNAs based on RNA-Seq data of mouse Klf1 knockout study
}

Lei Sun 1,2,3, Zhihua Zhang ${ }^{2}$, Timothy L Bailey ${ }^{3}$, Andrew C Perkins ${ }^{4}$, Michael R Tallack ${ }^{4}$, Zhao Xu ${ }^{1}$ and Hui Liư ${ }^{1 *}$

\begin{abstract}
Background: Study on long non-coding RNAs (IncRNAs) has been promoted by high-throughput RNA sequencing (RNA-Seq). However, it is still not trivial to identify IncRNAs from the RNA-Seq data and it remains a challenge to uncover their functions.

Results: We present a computational pipeline for detecting novel IncRNAs from the RNA-Seq data. First, the genomeguided transcriptome reconstruction is used to generate initially assembled transcripts. The possible partial transcripts and artefacts are filtered according to the quantified expression level. After that, novel IncRNAs are detected by further filtering known transcripts and those with high protein coding potential, using a newly developed program called IncRScan. We applied our pipeline to a mouse KIf1 knockout dataset, and discussed the plausible functions of the novel IncRNAs we detected by differential expression analysis. We identified 308 novel IncRNA candidates, which have shorter transcript length, fewer exons, shorter putative open reading frame, compared with known protein-coding transcripts. Of the IncRNAs, 52 large intergenic ncRNAs (lincRNAs) show lower expression level than the protein-coding ones and 13 IncRNAs represent significant differential expression between the wild-type and KIf1 knockout conditions.

Conclusions: Our method can predict a set of novel IncRNAs from the RNA-Seq data. Some of the IncRNAs are showed differentially expressed between the wild-type and KIf1 knockout strains, suggested that those novel IncRNAs can be given high priority in further functional studies.
\end{abstract}

\section{Background}

The category of long non-coding RNAs (lncRNAs) is composed of non-coding RNAs (ncRNAs) with long transcript length (> 200 nucleotides) [1]. The lncRNAs may carry out a variety of functions, e.g. scaffolding multiple proteins to form a complex, and regulating gene expression [2-11], however, most lncRNAs' functions remain to be specified. During the past decade, a growing number of newly detected lncRNAs have been reported thanks to the development of relevant biotechnology and computational methods [4,12-16]. Early tiling microarrays were used to detect the lncRNAs in the mammalian transcriptome $[4,5]$, however, they could not detect precise gene

\footnotetext{
*Correspondence: Ihcumt@hotmail.com

${ }^{1}$ School of Information and Electrical Engineering, China University of Mining and Technology, Xuzhou, JiangSu 221008, PR China

Full list of author information is available at the end of the article
}

structures and exon linkages of the lncRNAs [14]. Subsequently, this problem was tackled by high-throughput RNA sequencing (RNA-Seq), which presented its advantage of revealing the whole transcriptome [17], including detailed gene structures and expression levels. So far, the RNA-Seq has been the major biotechnology for lncRNA study [13]. For example, by using RNA-Seq, Guttman et al. [14] obtained detailed information of over a thousand large intergenic ncRNAs (lincRNAs) in three mouse cell types [14].

However, studying lncRNAs based on RNA-Seq encounters several technical problems. First, the assembled transcriptome may include partial transcripts and artefacts caused by RNA-Seq problems, such as low sequencing depth, sequencing biases [18] and short read alignment errors [19]. For lowly expressed transcripts, the sequencing biases may introduce undesired gaps in the assembly, resulting in partially assembled 
transcripts [20], which may be mistakenly identified as lncRNAs. The similar mistakes could also be introduced by low sequencing depth for lowly expressed transcripts. Moreover, the incomplete and erroneous assemblies can affect downstream analysis $[21,22]$. Second, transcriptome reconstruction [23] based on RNA-Seq reads may produce a variety of transcripts, e.g. completely assembled transcripts, intronic RNAs [24] and antisense transcripts [16], which are classified by comparing to the known gene annotations. Thus it is not trivial to identify lncRNAs from such complex assemblies. Third, it is still difficult to distinguish the lncRNAs from the proteincoding mRNAs [1] or short peptides. A protein-coding mRNA can be defined by open reading frame (ORF) greater than 100 amino acids (aa) or 300 nucleotides (nt) [25], but this is arbitrary and incorrect [26]. Here we present a computational pipeline to address these problems.

Although thousands of lncRNAs have been identified $[13,14,16]$, only a handful of them were functionally characterized. Given the difficulty to experimentally characterize the biological functions of the lncRNAs [7], and given the growing body of genomics and epigenomics data becoming available relevant to lncRNAs' biological functions, it is interesting to predict lncRNAs' functions computationally. We applied our computational method to an RNA-Seq dataset derived from a Klf1 gene knockout study on mouse fetal liver tissue [27]. Previous studies based on the Klf1 knockout study manifested that Klf1 is the founding member of a family of 17 transcription factors in mammals [28]. Klf1 knockout mice die from anemia by embryonic day 15 (E15), with severe defects in differentiation, hemoglobinization, enucleation, and membranecytoskeleton organization of red blood cells [29]. However, very little is known of the lncRNAs regulated by Klf1 or that participate in the development of erythroid cells. Here, we recruit the differential expression analysis to explore the lncRNAs that may function in the erythropoiesis.

\section{Methods}

\section{Datasets}

The RNA-Seq dataset for the Klf1 knockout experiment on mouse embryonic day 14.5 (E14.5) fetal liver tissue can be obtained from NCBI Gene Expression Omnibus (GEO) [30] database with accession number GSE33979 [27], and it includes 6 replicates (3 for wild-type and 3 for Klf1 knockout) totalling 160 million 76-base singleend reads generated by Illumina GAIIx sequencing on polyadenylated selected (Poly- $A^{+}$) RNAs. Bowtie [31] index of Mus musculus genome (mm9), Ensembl [32] and NCBI reference sequences (RefSeq) mouse gene annotations [33] are all available on Cufflinks' website [34]. University of California Santa Cruz (UCSC) mouse known gene annotations [35] can be downloaded from the UCSC genome browser [36].

\section{Pipeline for predicting novel IncRNAs}

There are two parts in our pipeline for predicting novel lncRNAs from the RNA-Seq data (Figure 1).

\section{Initial assembly}

Initial assembly (Figure 1-a) represents a genome-guided strategy for transcriptome reconstruction [23]. The raw RNA-Seq reads were first mapped onto the $\mathrm{mm} 9$ genome by Tophat 2.0.3 [19]. After that, the un-mapped reads were trimmed to $50 \mathrm{nt}$ before re-mapping. The final mapped reads of each replicate include two parts, namely 'Mapped reads 1' and 'Mapped reads 2'. Moreover, the '-G' option of Tophat together with the Gene Transfer Format (GTF) file of the Ensembl gene annotation was used for read mapping. With the read alignments, we calculated the overlap ratio (OR) between the replicates of each condition (Additional file 1). To increase the read coverage, we merged the read alignments of all six replicates into one Binary version of Sequence Alingment/Map (BAM) using Samtools 0.1.18 [37]. Then the mapped reads were assembled by Cufflinks 2.0.2 [21]. In the transcriptome assembly, we performed Reference Annotation Based Transcript (RABT) assembly [38] with the RefSeq gene annotation to compensate incompletely assembled transcripts caused by read coverage gaps in the regions of RefSeq genes.

\section{Novel IncRNAs detection}

Novel lncRNAs detection (Figure 1-b) is aimed at detecting novel lncRNAs from the initial assemblies. Specifically, the initial assemblies were first compared to a set of combined gene annotations (See below) using cuffcompare [22]. As a result, not only the assemblies that completely match the annotations will be detected, but also the novel transcripts can be categorized into different categories according to their locations compared with the reference genes. Notably, only multi-exon transcripts were retained for the comparison and downstream processing. Then low-quality assemblies were filtered according to the optimum Fragments Per Kilobases of exon per Million fragments mapped (FPKM) [21] threshold (2.12, see below). After that, we used a newly-developed program called lncRScan (See below) to detect novel lncRNAs.

\section{Combined gene annotations of RefSeq, Ensembl and UCSC mouse known genes}

The cuffcompare program [22] was used to merge the RefSeq, Ensembl and UCSC mouse known genes into one set of gene annotation for comparing with the assembled transcripts. 


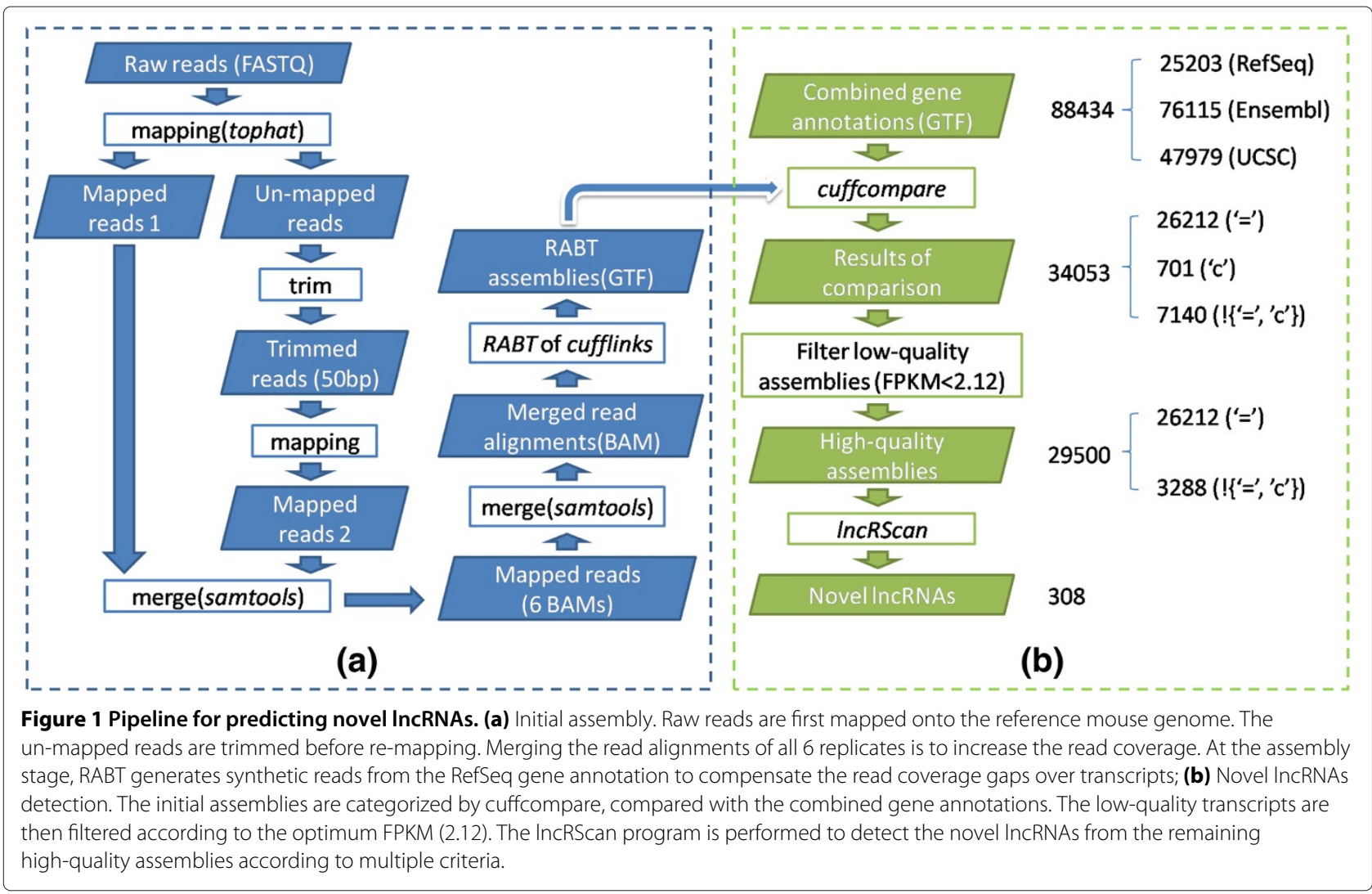

\section{FPKM threshold for classifying complete and partial transcripts}

Based on the merged read alignments, we conducted an experiment to evaluate the performance of FPKM in classifying complete and partial transcripts. Specifically, we first ran cufflinks on the merged read alignments with default options. Then the output assemblies with FPKM values estimated were categorized using cuffcompare, compared with the combined gene annotations. With the results, we evaluated the performance of different FPKM thresholds in classifying the complete and partial transcripts by Receiver Operating Characteristic (ROC) [39].

\section{Calculating optimum FPKM threshold}

The optimum FPKM threshold for classifying the complete and partial transcripts were calculated by training the FPKM values estimated from the experiment above. The index of the optimum FPKM threshold can be obtained by optimizing the sensitivity and specificity in classifying the complete and partial transcripts with formula 1 .

$$
i^{*}=\underset{i \in I}{\arg \min }\left\{\sqrt{(1-\text { sensitivities }[i])^{2}+(1-\text { specificities }[i])^{2}}\right\}
$$

where $i^{*}$ represents the index of the optimum FPKM threshold. On the right of formula 1 , sensitivities $[i]$ and specificities $[i]$ respectively denote the $i$ th sensitivities and specificities, given an index $i$. The $i$ is enumerated in $I$, ranging from 1 to the size of a FPKM threshold set. Then we can get the optimum FPKM threshold using formula 2.

$$
t^{*}=T\left[i^{*}\right]
$$

where $t^{*}$ denotes the optimum FPKM threshold. The FPKM threshold set $T$ were generated by pROC [39], given the FPKM values of the complete and partial transcripts.

\section{IncRScan}

To detect novel lncRNAs from a set of high-quality assemblies, a five-step program named long non-coding RNA Scan (lncRScan) was designed (Figure 2). Step 1 'extract_category' is used to extract five candidate categories of transcripts, including ' $i$ ', ' $j$ ', 'o', ' $u$ ' and ' $x$ ', which may contain novel lncRNAs. Specifically, the ' $i$ ' category may contain the lncRNAs falling entirely within an intron of known genes. And the ' $\mathrm{j}$ ' category may include alternative long non-coding isoforms of known genes as they share at least one spliced site with reference transcripts. The ' $u$ ' category may involve the intergenic lncRNAs (lincRNAs). The 'o' category may contain the lncRNAs having generic exonic overlap with a known transcript while the ' $x$ ' transcripts also have exonic overlap 


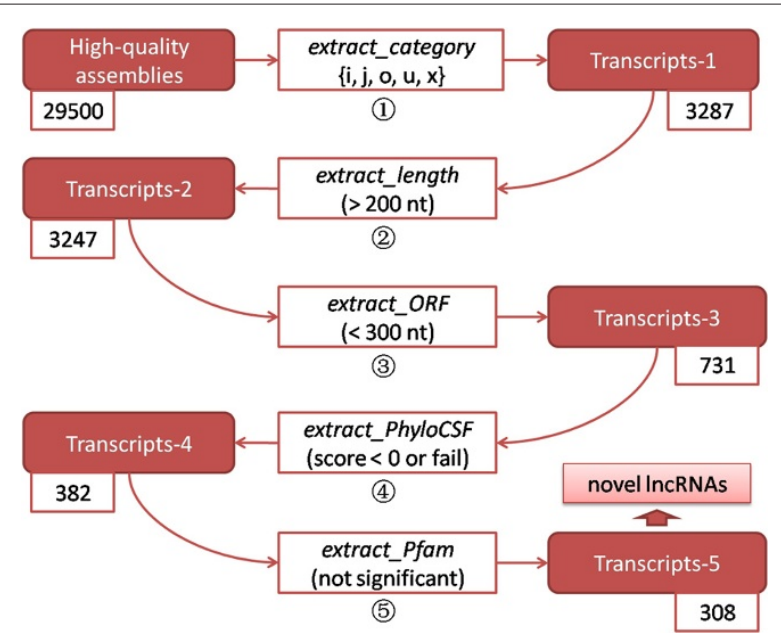

Figure 2 Steps of IncRScan. (1) 'extract_category' extracts five candidate categories of assemblies (Transcripts-1), including ' 'i', 'j', 'o', ' $u$ ' and ' $x$ '; (2) 'extract_length' is used to extract the transcripts with length $>200 \mathrm{nt}$ (Transcripts-2); (3) 'extract_ORF' selects the transcripts with maximum putative ORF < 300 nt (Transcripts-3); (4)

'extract_PhyloCSF' extracts the transcripts with PhyloCSF score $<0$ or test failure due to ORF $<25$ aa (Transcripts-4); (5) 'extract_Pfam'

searches the remaining transcripts in the Pfam database and excludes the transcripts with significant protein domain hits. Towards the end of IncRScan, the remaining 308 transcripts (Transcripts-5) are defined as the novel IncRNAs.

with reference but on the opposite strand. Therefore, the five categories defined here may include novel lncRNAs potentially. On the other hand, all categories of transcripts extracted have not been annotated by either of RefSeq, Ensembl and UCSC known genes, so the predicted lncRNAs can be 'novel'. Step 2 'extract length' is used to extract the transcripts having long exonic length (> $200 \mathrm{nt}$ ) according to the lncRNA's definition. Step 3 'extract_ORF' is set to exclude the assemblies that have long ( $\geq 300 \mathrm{nt}$ ) putative ORF. Then steps 4 and 5 are used to exclude the transcripts of protein-coding potential. In Step 4 'extract_PhyloCSF,' Phylogenetic Codon Substitution Frequency (PhyloCSF) [40] is recruited to filter the transcripts of protein-coding potential from an evolutionary view. Briefly, PhyloCSF conducts a comparative genomics method for classifying protein-coding and non-coding sequences [40]. Since the sequence alignments are required for running PhyloCSF, we used Galaxy [41-43] to 'stitch' 29 mammalian alignments according to the input transcripts. In Step 5 'extract_Pfam', the amino acid sequences of the remaining transcripts are searched in Pfam [44] (both Pfam-A and Pfam-B) for comparing to known proteins or protein domains, and the transcripts with significant domain hits are excluded.

To evaluate the performance of lncRScan in identifying lncRNAs or filtering mRNAs, we ran the steps 3-5 of lncRScan on four datasets respectively. The first dataset (D-1) contains 1615 multi-exon RefSeq ncRNAs with length $>200 \mathrm{nt}$ and the second one (D-2) records 1615 mRNAs randomly sampled from 26368 RefSeq mRNAs. The other two datasets (D-3 and D-4) include 3230 and 4845 mRNAs sampled from the RefSeq mRNAs respectively. The numbers of the retained and filtered transcripts through the steps 3-5 of lncRScan are summarized in Table 1. We can see that 771 (47.74\%) lncRNAs of D-1 were retained after the steps 3-5. In contrast, most (99.6\%99.7\%) of the mRNAs (D-2, D-3 and D-4) were filtered by the steps 3-5. The result indicates that the filters of lncRScan can dramatically reduce the number of mRNAs. Notably, the step 3 adopting the ORF threshold can filter a large proportion of mRNAs thereby alleviating the overload of PhyloCSF and Pfam calculation. However, some true lncRNAs were filtered through the pipeline, which made the final lncRNAs prediction much stringent.

In addition, IncRScan is available to the scientific community and it can be obtained by svn checkout http:// lncrscan.googlecode.com/svn/trunk/lncrscan-read-only. Other details about lncRScan can be found on http:// code.google.com/p/lncrscan/.

\section{Differential expression analysis}

The cuffdiff [22] program was performed to conduct differential expression (DE) tests between the wild-type (WT) and Klf1 knockout (Klf1 KO) samples (Figure 3). The fold changes were calculated via $\log _{2} \frac{F P K M W T}{F P K M \text { Klf } 1 K O}$. A transcript will be reported $\mathrm{DE}$ significant if the test gives that the FDR-adjusted p-value after Benjamini-Hochberg correction [45] for multiple-testing represent statistical significant (q-value $<0.05)[46]$.

\section{Comparisons of transcript length, exon number, ORF length and expression level}

The novel lncRNAs we detected were compared to 26368 RefSeq protein-coding transcripts ('NM' prefix) and 2843 RefSeq non-coding transcripts ('NR' prefix) in terms of transcript length, exon number and ORF length. Since a real ncRNA does not have an ORF, a putative ORF of the ncRNA candidate is defined by the longest consecutive codon chain of the ncRNA candidate for comparing with the protein-coding genes. Moreover, for both of the WT and Klf1 KO conditions, we compared the quantified expression levels (FPKM) of the novel lncRNAs to that of the known protein-coding transcripts, which were extracted from the RefSeq and Ensembl gene annotations. The novel lncRNAs and protein-coding transcripts used for FPKM comparison all have enough expression levels $(\mathrm{FPKM} \geq 2.12)$.

\section{Results}

\section{Initially assembled transcripts}

We started our analysis with short read mapping (Figure 1-a), and approximately 138 million reads were 
Table 1 Numbers of retained and filtered transcripts through steps 3-5 of IncRScan

\begin{tabular}{|c|c|c|c|c|c|c|}
\hline \multirow[t]{2}{*}{ Test data } & \multicolumn{2}{|c|}{ extract_ORF(Step 3) } & \multicolumn{2}{|c|}{ extract_PhyloCSF(Step 4) } & \multicolumn{2}{|c|}{ extract_Pfam(Step 5) } \\
\hline & Retained & Filtered & Retained & Filtered & Retained & Filtered \\
\hline D-1 (1615 IncRNAs) & $952(58.95 \%)$ & 663 & $813(50.34 \%)$ & 139 & $771(47.74 \%)$ & 42 \\
\hline D-2 (1615 mRNAs) & $33(2 \%)$ & 1582 & $12(0.74 \%)$ & 21 & $6(0.37 \%)$ & 6 \\
\hline D-3 (3230 mRNAs) & $89(2.76 \%)$ & 3141 & $45(1.44 \%)$ & 44 & $10(0.31 \%)$ & 35 \\
\hline D-4 (4845 mRNAs) & $112(2.31 \%)$ & 3733 & $50(1.03 \%)$ & 62 & $18(0.37 \%)$ & 32 \\
\hline
\end{tabular}

successfully mapped onto the mm9 genome (Table 2). With the merged alignments of six replicates, 34053 multi-exon transcripts (26212 annotated, 701 contained by annotations and 7140 novel potentially) were assembled in total, compared with 88434 transcripts of the combined gene annotations. Then we obtained the categories of the initial assemblies by comparing to the combined gene annotations (Table 3). It is notable that the initial assemblies include several categories of transcripts, e.g. transcripts that have complete match intron chain compared with known genes ('=' classcode) and those contained by known genes ('c' classcode). Of the initial assemblies, 26212 (76.97\%) transcripts have been annotated by either of RefSeq, Ensembl and UCSC known genes.

\section{Filtering low-quality assemblies with optimum FPKM threshold}

FPKM can unbiasedly represent quantified expression level of an assembled transcript, and it can be estimated by maximum likelihood estimation (MLE) under a statistical model of cufflinks [21], which also corrects sequencing biases [18] in the estimation. Figure 4 shows the FPKM distributions [47] of the complete ('=' classcode) and partial ('c' classcode) transcripts assembled from the experiment of FPKM threshold (See Methods) while Figure 5 shows the corresponding ROC curve. Notably, the complete transcripts represent much larger FPKM than the partial ones on average $\left(\sim 29.67\right.$ vs $\sim 4.86, P<2.2 \times 10^{-16}$,

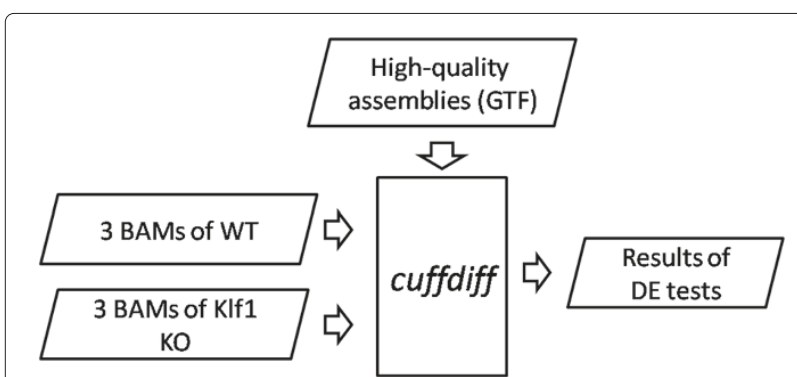

Figure 3 Differential expression tests. The cuffdiff program performs differential expression tests between the WT and KIf1 KO samples based on the read alignments (BAM) of the six replicates and high-quality assemblies (GTF).
Welch Two Sample t-test). According to the significant difference of FPKM distributions of complete and partial assemblies, we calculated the optimum FPKM threshold (2.12) based on our data (See Methods). We assumed that the artificial transcripts represent either similar FPKM distribution to the partial transcripts or lower FPKM than the partial ones, thus the optimum threshold can be used to filter both of the partial assemblies and artefacts from the 7140 novel assemblies.

\section{Identification of high-quality assemblies}

We pooled a set of high-quality assemblies (Additional file 2) for downstream analysis. The high-quality assemblies consist of two categories. One category contains the 26212 initial assemblies that completely match the combined gene annotations ("=' classcode). The other category refers to the 3288 transcripts extracted from the 7140 novel assemblies $\left(!\left\{{ }^{\prime}=,{ }^{*}\right.\right.$ ' $\left.\}\right)$, which satisfy the expression criterion (FPKM $\geq 2.12$ ).

\section{Novel mouse embryonic IncRNAs}

We applied our newly developed lncRNAs detector lncRScan to the high-quality assemblies and detected 308 novel mouse embryonic lncRNAs (Additional file 3). The novel lncRNAs were further classified into 5 categories by comparing with the known gene annotations (Table 4). Specifically, 52 lncRNAs were assigned the ' $u$ ' classcode since they were located in the intergenic regions. And 26 lncRNAs with the 'i' classcode fall entirely within the intron of known genes. The other lncRNAs all have exon overlap with known genes. Specifically, 44 lncRNAs with the 'o' classcode have generic exonic overlap with

Table 2 Read mapping summary

\begin{tabular}{lccc}
\hline Replicate & Raw reads & Un-mapped & Mapped \\
\hline KO_1 & 25153995 & $5713351(22.7 \%)$ & $19440644(77.3 \%)$ \\
KO_2 & 26269828 & $3294901(12.5 \%)$ & $22974927(87.5 \%)$ \\
KO_3 & 25988788 & $6032342(23.2 \%)$ & $19956446(76.8 \%)$ \\
WT_1 & 20034326 & $2006957(10.0 \%)$ & $18027369(90.0 \%)$ \\
WT_2 & 22221706 & $4486281(20.2 \%)$ & $17735425(79.8 \%)$ \\
WT_3 & 45034903 & $4678496(10.4 \%)$ & $40356407(89.6 \%)$ \\
total & 164703546 & $26212328(15.9 \%)$ & $138491218(84.1 \%)$ \\
\hline
\end{tabular}


Table 3 Categories of initial assemblies

\begin{tabular}{lccl}
\hline Class code & Transcript number & Percentage & Description \\
\hline$=$ & 26212 & $76.97 \%$ & Complete match of intron chain \\
$\mathrm{c}$ & 701 & $2.06 \%$ & Contained by a reference transcript \\
$\mathrm{j}$ & 6207 & $18.23 \%$ & At least one splice junction is shared with a reference transcript \\
$\mathrm{i}$ & 155 & $0.46 \%$ & A transfrag falling entirely within a reference intron \\
$\mathrm{O}$ & 187 & $0.55 \%$ & Generic exonic overlap with a reference transcript \\
$\mathrm{u}$ & 492 & $1.44 \%$ & Unknown, intergenic transcript \\
$\mathrm{x}$ & 98 & $0.29 \%$ & Exonic overlap with reference on the opposite strand \\
$\mathrm{s}$ & 1 & $0.00 \%$ & An intron of the transfrag overlaps a reference intron on the oppo- \\
& & & site strand \\
total & 34053 & $100 \%$ & Total
\end{tabular}

known genes and 6 ' $x$ ' lncRNAs also have exonic overlap with known genes but on the opposite strand. The 180 lncRNAs with 'j' can be long non-coding isoforms of known genes. In addition, the 308 novel lncRNAs we predicted were compared with 36991 ones annotated by NONCODE 3.0 [48]. Of the 308 novel lncRNAs, 5 (1.62\%) ones have the same structure as NONCODE lncRNAs (Additional file 1) and another 75 (24.35\%) ones partially overlap the NONCODE lncRNAs (Figure 6). By excluding the 80 lncRNAs that overlap the NONCODE annotation, we can get a more stringent set of novel lncRNAs.

Novel IncRNAs have shorter transcript length, fewer exons and shorter putative ORF than protein-coding transcripts

Previous studies in mammals have shown that lncRNAs are shorter in length and fewer in exon number than are protein-coding transcripts $[13,14,16]$. To determine whether the embryonic lncRNAs we detected have the same features, we compared the 308 novel lncRNAs to not only 26368 protein-coding transcripts, but also 2843 known non-coding ones, annotated by RefSeq (See Methods). As shown in Figure 7, the novel lncRNAs represent much shorter transcript length on average than either RefSeq protein-coding $(\sim 1.2 \mathrm{~kb}$ vs $\sim 3.1 \mathrm{~kb}$, $P<2.2 \times 10^{-16}$, Welch Two Sample t-test) or non-coding transcripts $\left(\sim 1.2 \mathrm{~kb}\right.$ vs $\left.\sim 1.9 \mathrm{~kb}, P=6.027 \times 10^{-14}\right)$ while the lncRNAs also show fewer exons than either of the RefSeq protein-coding $\left(\sim 2.8\right.$ vs $\left.\sim 10.0, P<2.2 \times 10^{-16}\right)$ and non-coding transcripts $\left(\sim 2.8\right.$ vs $\left.\sim 3.3, P=5.096 \times 10^{-8}\right)$, agreed with a previous report [13]. In addition, we also compared the putative ORF lengths of the lncRNAs to that of the RefSeq genes (both protein-coding and noncoding). As a result, the novel lncRNAs represent shorter putative ORF length than either RefSeq protein-coding RNAs $\left(\sim 0.17 \mathrm{~kb}\right.$ vs $\left.\sim 1.6 \mathrm{~kb}, P<2.2 \times 10^{-16}\right)$ or ncRNAs $\left(\sim 0.17 \mathrm{~kb}\right.$ vs $\left.\sim 0.30 \mathrm{~kb}, P<2.2 \times 10^{-16}\right)$,

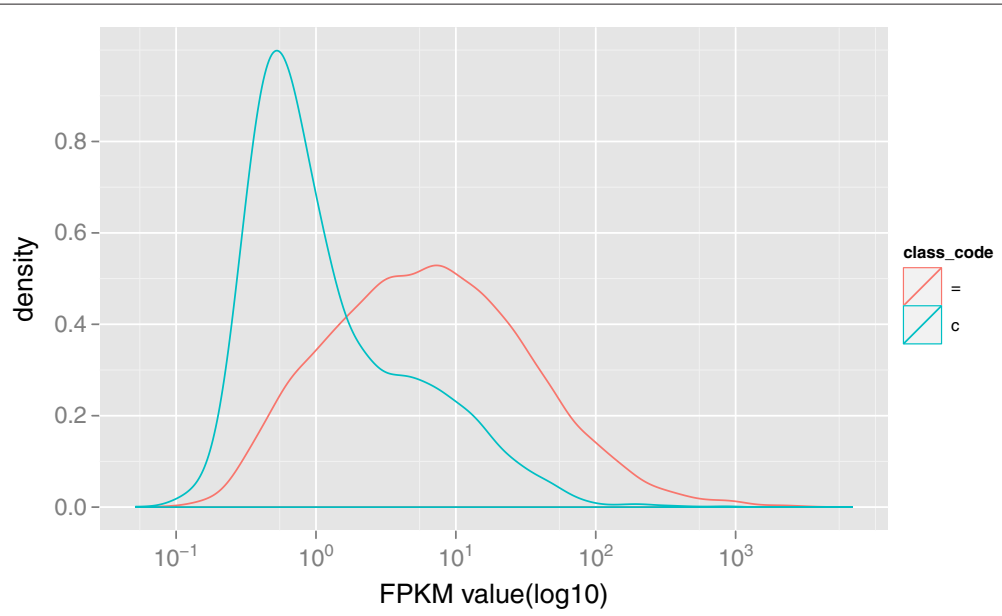

Figure 4 FPKM distributions of complete and partial transcripts. The ${ }^{\prime}={ }^{\prime}$ classcode is originally assigned to the transcripts that have complete match intron chain with a reference transcript and they can be treated as complete transcripts while the ' $c$ ' classcode is attached to the transcripts contained by reference and they are defined as partial assemblies. The complete (' $=$ ', red curve) and partial (' $c$ ', blue curve) transcripts assembled from the read alignments represent distinguishable FPKM distributions from each other ( 29.67 vs 4.86). 


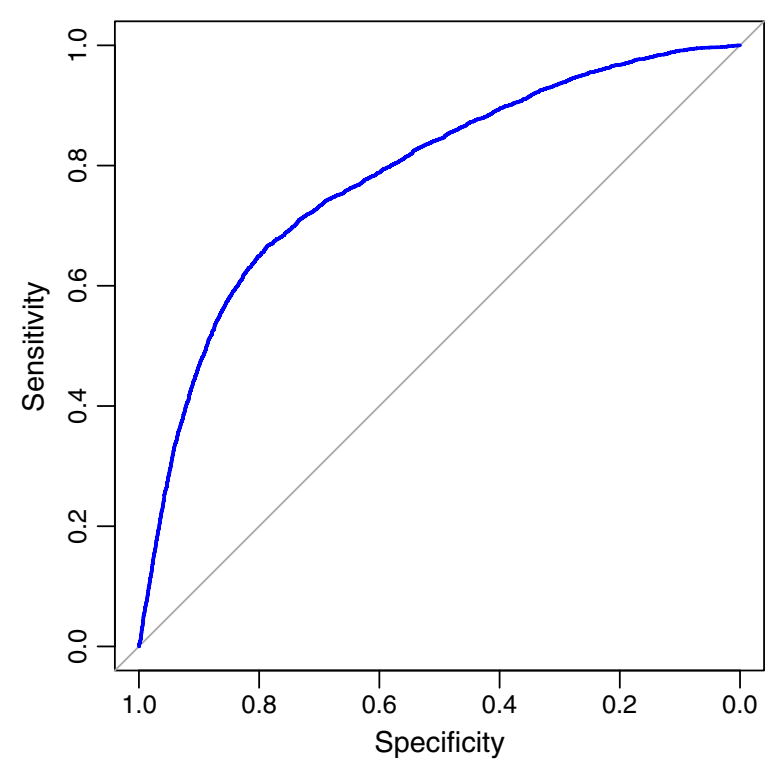

Figure 5 Performance of FPKM in distinguishing between complete and partial transcripts. An assembled transcript will be classified into the category of complete assemblies (' $=$ ' classcode) if its FPKM is larger than a given threshold, otherwise it will be put into the partial category (' $c$ ' classcode). The blue ROC curve [39] represents the performance of FPKM in classifying the complete and partial transcripts. The corresponding Area Under Curve (AOC) is 0.7825 .

consistent with a previous report on zebrafish embryonic lncRNAs [16]. Although the novel lncRNAs candidates are to be ncRNAs, they can differ from the RefSeq ncRNAs used for comparison in some features due to several reasons as follows. First, the RefSeq ncRNAs do not only include lncRNAs, but also other categories of ncRNAs, e.g. microRNAs and small nucleolar RNAs. Second, the lncRNAs can be further classified according to their biological functions, thus the features of different categories of lncRNAs may differ from each other. The lncRNAs we detected may not come from the same category as that annotated by RefSeq. Third, the unbalanced population sizes can affect the comparison between the two categories of ncRNAs. Last, the putative ORF length of the lncRNAs we predicted were limited $(<300 \mathrm{nt})$, which can affect the ORF comparison. Therefore it is reasonable to see that the two categories of ncRNAs repre- sent slight statistical difference, which is far less than that between the mRNAs and ncRNAs.

\section{Novel lincRNAs have lower expression level than protein-coding transcripts}

Previous studies also showed that lncRNAs are expressed at significantly lower levels than are protein-coding transcripts $[13,14,16]$. To determine whether the embryonic lncRNAs we detected have the same expression feature, we compared the quantified expression levels (FPKM) of the 308 novel lncRNAs to that of the known proteincoding transcripts (Figure 8). In the WT condition (Figure 8-a), the protein-coding transcripts represents slightly higher expression than the novel lncRNAs on average ( $\sim 50.92$ vs $\sim 44.54, P=0.554$, Welch Two Sample t-test). Similarly, in the Klf1 KO condition (Figure 8-b), the protein-coding transcripts also show slightly higher expression than the lncRNAs on average $(\sim 37.63$ vs $\sim 34.06, P=0.6986)$. The comparison result indicates that the total novel lncRNAs do not show significant lower expression than the protein-coding ones. Moreover, we extracted the 52 lincRNAs ('u' classcode) from the 308 lncRNAs for the expression comparison. The result manifests that the lincRNAs we predicted represents significant lower expression than the protein-coding ones in either WT or Klf1 KO condition $(\sim 11.29$ vs $\sim 50.93$, $P<2.2 \times 10^{-16}$, and $\sim 9.38$ vs $\sim 37.63, P<2.2 \times 10^{-16}$, respectively).

\section{Differentially expressed IncRNAs}

Using cuffdiff, we conducted the differential expression (DE) tests between the WT and Klf1 KO samples for analysing the function of the novel lncRNAs. At the gene level (Figure 9-a), Klf1 represents like an activator since more assembled genes are significantly repressed (334) after Klf1 is knocked out than the activated ones (250). At the transcript level (Figure 9-b), Klf1 also behaves like an activator since more transcripts are significantly repressed (262) after Klf1 is knocked out than the activated ones (147). Moreover, we detected 13 (Additional file 4) novel lncRNAs with DE significant. Notably, Klf1 still functions like an activator for the 13 nncRNAs (10 repressed vs 3 activated after Klf1 is knocked out, Figure 9-c). Thus it

Table 4 Categories of novel IncRNAs

\begin{tabular}{lccl}
\hline Class code & Transcript number & Percentage & Description \\
\hline $\mathrm{j}$ & 180 & $58.44 \%$ & At least one splice junction is shared with a reference transcript \\
$\mathrm{i}$ & 26 & $8.44 \%$ & A transfrag falling entirely within a reference intron \\
$\mathrm{O}$ & 44 & $14.29 \%$ & Generic exonic overlap with a reference transcript \\
$\mathrm{u}$ & 52 & $16.88 \%$ & Unknown, intergenic transcript \\
$\mathrm{x}$ & 6 & $1.95 \%$ & Exonic overlap with reference on the opposite strand \\
total & 308 & $100 \%$ & Total \\
\hline
\end{tabular}




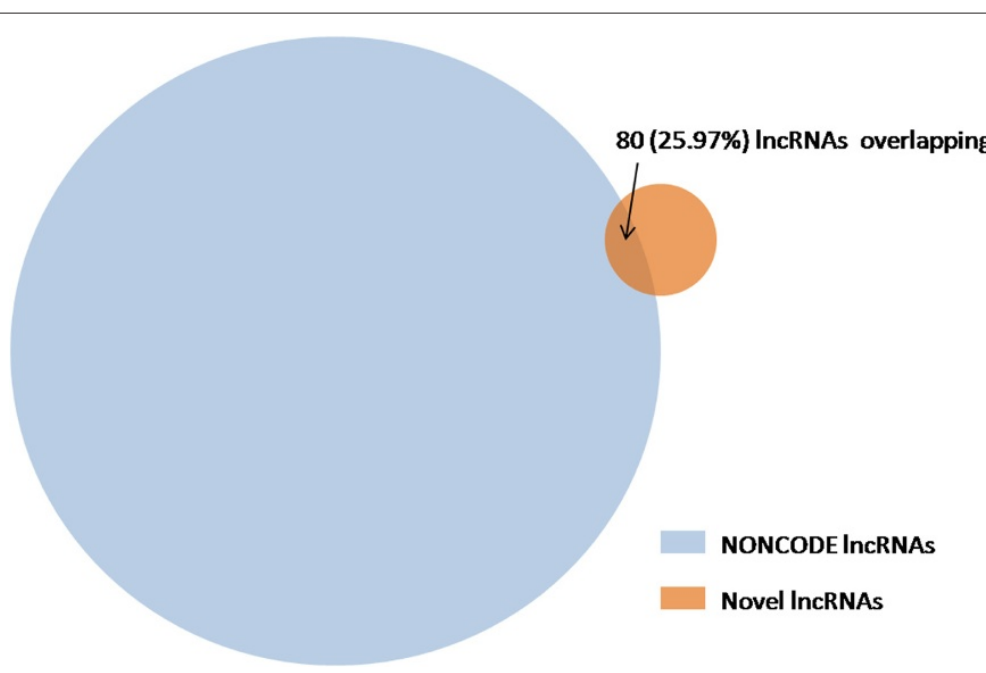

Figure 6 Comparison between novel IncRNAs and NONCODE IncRNAs. There are 36991 IncRNAs annotated by NONCODE 3.0 and 308 IncRNAs predicted by our method. Of the 80 (25.97\% of our prediction) overlapped IncRNAs, 5 ones have been exactly annotated by NONCODE 3.0.

is obvious that Klf1 can function as an activator globally, regulating the expression of a number of genes or transcripts including the IncRNAs we detected. The detailed categories of the 13 IncRNAs of DE significant can be seen from Table 5 .
However, cuffdiff does a length correction that has a tendency to inflate the FPKM counts for small transcripts, which can interfere the differential expression analysis. To alleviate this problem, we re-ran the DE tests with the "no-effective-length-correction" parameter. As a result, we

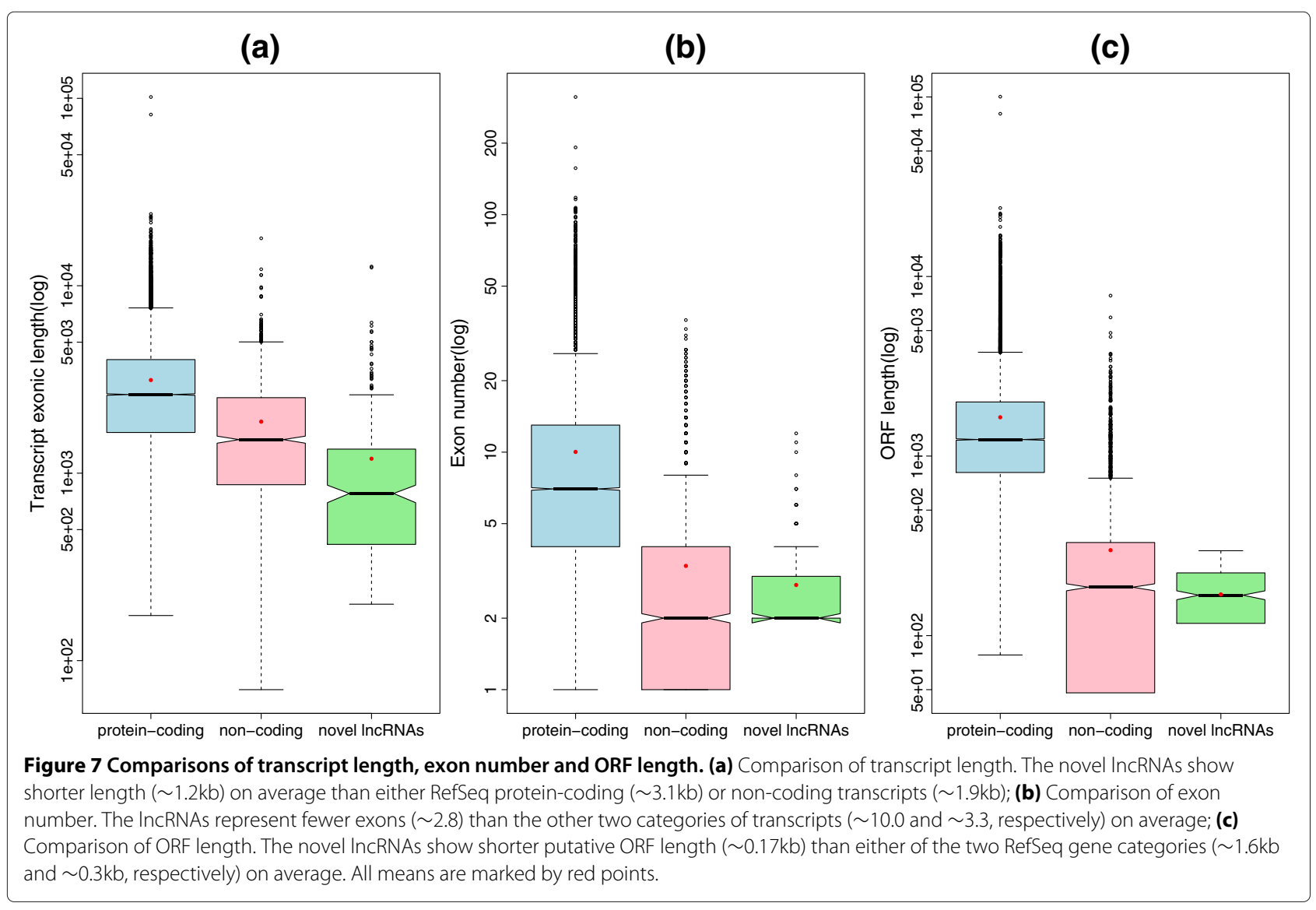




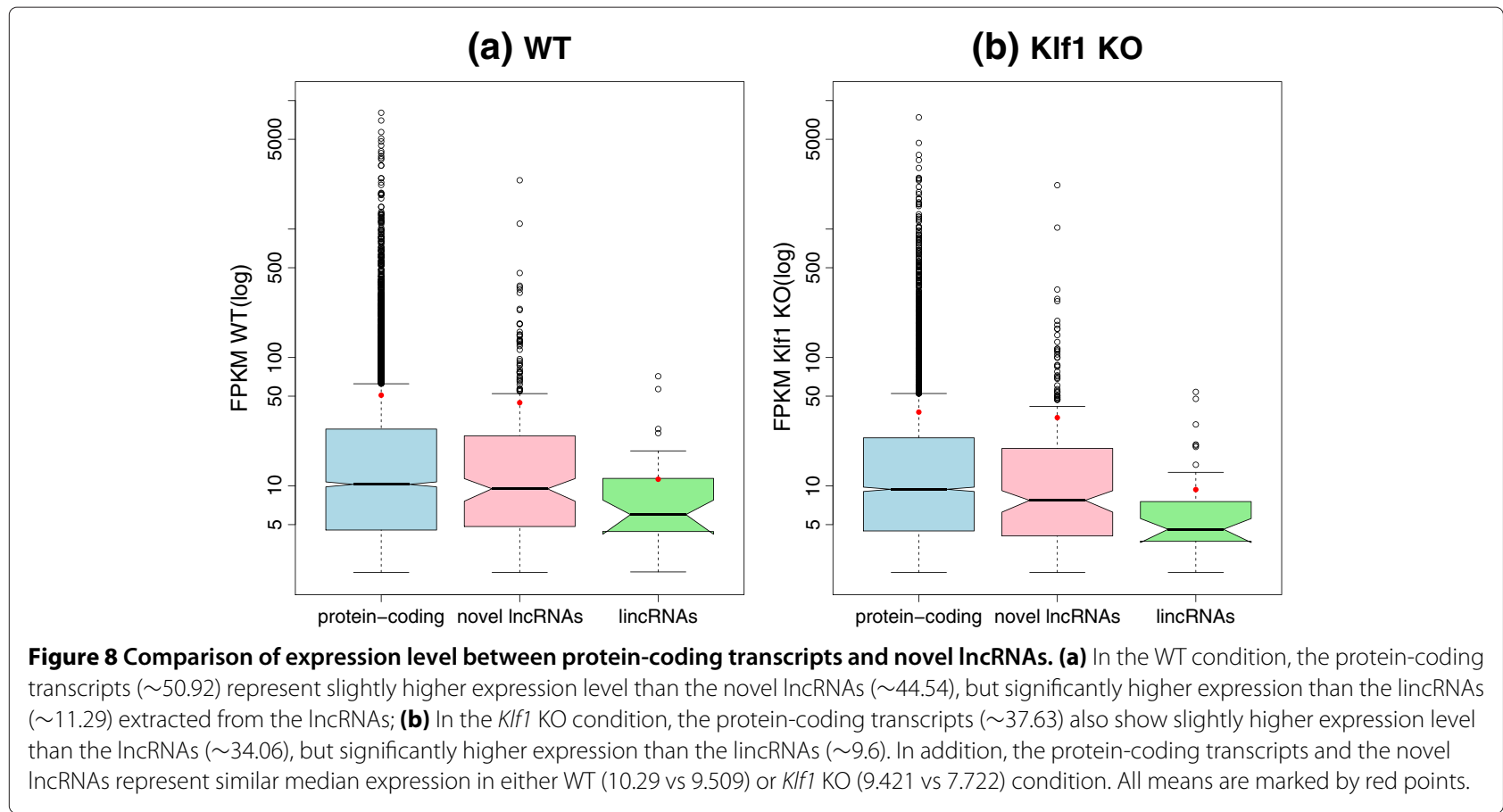

obtained the same results as that without the parameter, which represent the robustness of our predictions.

\section{Discussion}

RNA-Seq has been revolutionizing the transcriptome study as it can effectively capture the whole transcriptome of various cell types under different conditions. Here we predicted 308 novel mouse embryonic lncRNAs from the RNA-Seq data of WT and Klf1 KO samples using a computational pipeline. The novel lncRNAs we detected represent shorter transcript length, fewer exons and shorter putative ORF length, and the 52 lincRNAs of the lncRNAs show lower expression level, compared with known protein-coding transcripts. Moreover, we identified 13 differentially expressed novel lncRNAs, which may be regulated by Klf1 and play functional roles in the development of erythroid cells potentially. Notably, two lncRNAs (IDs: 2_00016377 and 2_00016378) we predicted
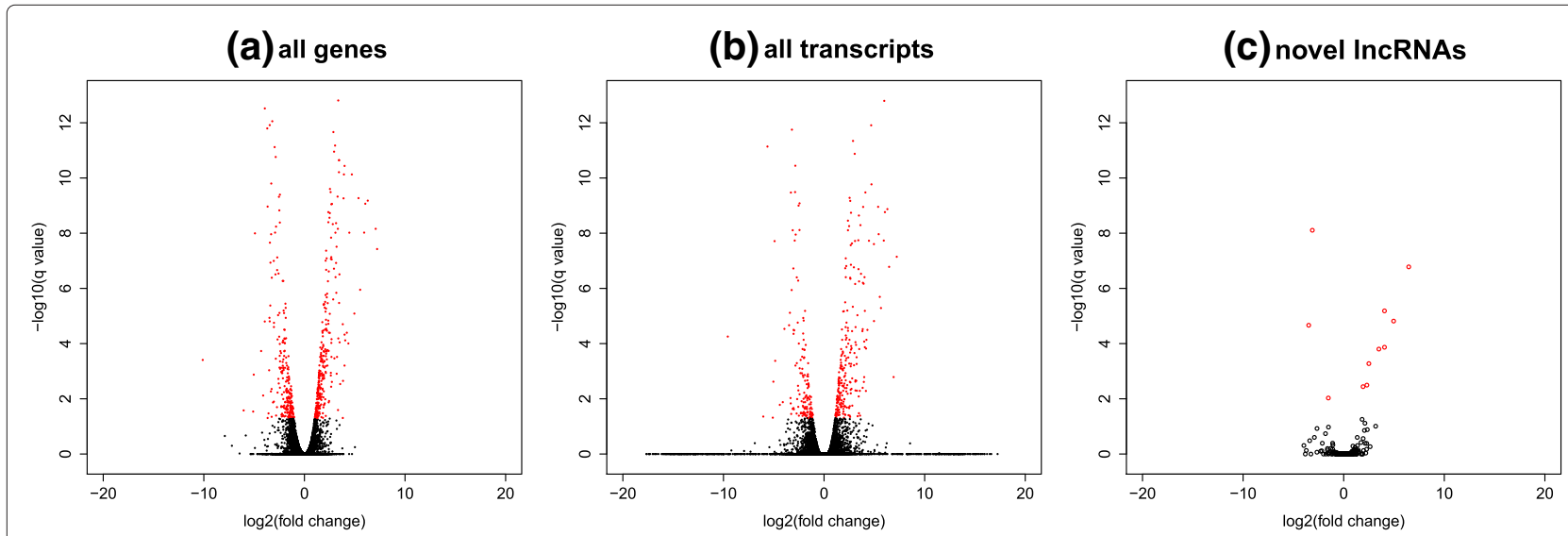

Figure 9 Differential expression of transcripts between WT and KIf1 KO. The three volcano plots illustrate the differential expression (DE) between the WT and KIf1 KO samples at either gene or transcript level: (a) DE of all genes. At the gene level, KIf1 globally appears to be an activator since more genes are significantly repressed (334, red points over the positive $x$-axis) than the activated ones (250, red points over the negative x-axis) after KIf1 is knocked out; (b) DE of all transcripts. At the transcript/isoform level, KIf1 also behaves like an activator since more transcripts are significantly repressed (262) than activated ones (147) after KIf1 is knocked out; (c) DE of the novel IncRNAs. For the 13 DE significant IncRNA transcripts, KIf1 still functions like an activator since 10 IncRNAs are repressed and 3 ones are activated after KIf1 is knocked out. The DE significant transcripts are all represented by red points. 
Table 5 Categories of novel IncRNAs of differential expression significant

\begin{tabular}{lccl}
\hline Class code & Transcript number & Percentage & Description \\
\hline $\mathrm{j}$ & 5 & $38.46 \%$ & At least one splice junction is shared with a reference transcript \\
$\mathrm{i}$ & 1 & $7.69 \%$ & A transfrag falling entirely within a reference intron \\
0 & 2 & $15.38 \%$ & Generic exonic overlap with a reference transcript \\
$\mathrm{u}$ & 4 & $30.77 \%$ & Unknown, intergenic transcript \\
$\mathrm{x}$ & 1 & $7.69 \%$ & Exonic overlap with reference on the opposite strand \\
total & 13 & $100 \%$ & Total \\
\hline
\end{tabular}

represent almost the same structures as another two lncRNAs predicted by Tallack et al. [27] based on the same dataset. Specifically, most exons of 2_00016377 and 2_00016378 match that of their 'lincred1-giant' and 'lincred1-dwarf' lncRNAs respectively. The slight difference may be caused by both of the strategies of transcriptome reconstruction and program versions used. Despite of that, the differential expression of the two lncRNAs we detected can be explained by Tallack et al's validation using Real-time Quantitative PCR (qRT-PCR) [49] on their 'lincred1' lncRNAs.

On the other hand, our pipeline followed a similar strategy for predicting human lincRNAs [13], but we differ in three aspects. First, we used FPKM as a feature for filtering low quality assemblies instead of the read coverage [13] due to the fact that FPKM can unbiasedly represent the expression level of a transcript and the read coverage does not show better performance than FPKM in classifying the complete and partial transcripts assembled from our data (AUCs are equal). Second, we excluded the transcripts having long putative ORF length ( $\geq 300 \mathrm{nt}$ ), which was previously used by the FANTOM consortium [50]. This arbitrary cutoff makes our predictions more stringent, but it must omit the lncRNAs having long putative ORF ( $\geq 300 \mathrm{nt}$ ). Last, we detected several DE significant IncRNAs, which composed a subset of the total lncRNAs we detected and they are more worth being investigated by loss and gain of function studies than the other novel lncRNAs in our scenario. Consequently, our computational methods can effectively alleviate further experimental work for studying the lncRNAs that may participate in the development of erythroid cells.

Although our method presented its ability in detecting novel lncRNA candidates, its prediction accuracy can be improved from several aspects, such as using more reliable reads generated by high-quality deep sequencing, paired-end sequencing and strand-specific sequencing. And recent single-molecule sequencing technologies can provide more unbiased ways to capture the transcriptome [51]. The sensitivity of transcriptome reconstruction can also be improved by using various strategies, such as integrating assembly results from Scripture [14]. In addition, the novel lncRNAs predicted from our computational pipeline should be validated by biological experiments, such as cloning and PCR-based techniques [22] as several ones have been tested in the original study by Tallack et al. [27]. Furthermore, additional genetic and/or epigenetic data sources, e.g. Chromatin Immunoprecipitation-Sequencing (ChIP-Seq) on chromatin signatures, can be valuable sources providing useful information for characterizing functions of the novel lncRNAs. And the loss and gain of function studies can be conducted for exploring regulatory mechanisms of the lncRNAs.

\section{Conclusions}

We predicted a set of novel lncRNAs using our computational pipeline from the RNA-Seq data of Klf1 knockout study, and the DE significant lncRNAs are worth being further studied with regard to their biological functions.

\section{Additional files}

Additional file 1: Supplementary materials. It contains supplementary materials supporting the main text.

Additional file 2: GTF of high-quality assembled transcripts. It is a GTF file recording the 28963 high-quality assemblies, which were used in the differential expression tests. And their structures can be visualized by UCSC genome browser.

Additional file 3: GTF of novel IncRNAs. It is a GTF file recording the 308 novel IncRNAts detected by IncRScan. The transcript structures can be visualized by UCSC genome browser.

Additional file 4: GTF of differentially expressed IncRNAs. It is a GTF file recording the 13 differentially expressed IncRNAs between the WT and KIf1 KO samples. The transcript structures can be visualized by UCSC genome browser.

\section{Competing interests}

The authors declare that they have no competing interests.

\section{Authors' contributions}

LS designed and implemented the method. ACP and MRT provided the raw datasets. LS carried out the experiments and analysis. LS, ZZ, TLB, ZX and HL wrote the paper. All authors read and approved the final manuscript.

\section{Acknowledgements}

The authors would like to acknowledge Fabian A. Buske and other members of the Bailey's group at IMB of the University of Queensland for helpful discussions. The authors would like to thank the reviewers giving helpful suggestions on the paper. This work was supported by China Postdoctoral 
Science Foundation 2012M511335 and 2012M511336, The Central Special Fund for Operating Expenses of College Basic Research 2010QNA47 and 2010QNA50 and Fok Ying-Tung Education Foundation for Young Teachers (121066). LS was partially supported by China Scholarship Council (CSC) scholarship.

\section{Author details}

${ }^{1}$ School of Information and Electrical Engineering, China University of Mining and Technology, Xuzhou, JiangSu 221008, PR China. ${ }^{2}$ Center for Computational Biology, and Laboratory of Disease Genomics and Personalized Medicine, Beijing Institute of Genomics, Chinese Academy of Sciences, No.7 Beitucheng West Road, Chaoyang District, Beijing 100029, PR China. ${ }^{3}$ Institute for Molecular Bioscience, The University of Queensland, Brisbane, Queensland 4072, Australia. ${ }^{4}$ Mater Medical Research Institute, Mater Hospital, Brisbane, Queensland 4101, Australia.

Received: 10 July 2012 Accepted: 4 December 2012

Published: 13 December 2012

References

1. Mercer TR, Dinger ME, Mattick JS: Long non-coding RNAs: insights into functions. Nat Rev Genet 2009, 10(3):155-159. [10.1038/nrg2521].

2. Amaral PP, Dinger ME, Mercer TR, Mattick JS: The Eukaryotic Genome as an RNA Machine. Science 2008, 319(5871):1787-1789.

3. Baker M: Long noncoding RNAs: the search for function. Nat Meth 2011, 8(5):379-383. [10.1038/nmeth0511-379].

4. Kapranov P, Cheng J, Dike S, Nix DA, Duttagupta R, Willingham AT, Stadler PF, Hertel J, Hackermüller J, Hofacker IL, Bell I, Cheung E, Drenkow J, Dumais E, Patel S, Helt G, Ganesh M, Ghosh S, Piccolboni A, Sementchenko V, Tammana H, Gingeras TR: RNA Maps Reveal New RNA Classes and a Possible Function for Pervasive Transcription. Science 2007, 316(5830):1484-1488.

5. Bertone P, Stolc V, Royce TE, Rozowsky JS, Urban AE, Zhu X, Rinn JL, Tongprasit W, Samanta M, Weissman S, Gerstein M, Snyder M: Global Identification of Human Transcribed Sequences with Genome Tiling Arrays. Science 2004, 306(5705):2242-2246.

6. Rinn JL, Kertesz M, Wang JK, Squazzo SL, Xu X, Brugmann SA, Goodnough LH, Helms JA, Farnham PJ, Segal E, Chang HY: Functional Demarcation of Active and Silent Chromatin Domains in Human HOX Loci by Noncoding RNAs. Cell 2007, 129(7):1311-1323.

7. Guttman M, Donaghey J, Carey BW, Garber M, Grenier JK, Munson G, Young G, Lucas AB, Ach R, Bruhn L, Yang X, Amit I, Meissner A, Regev A, Rinn JL, Root DE, Lander ES: lincRNAs act in the circuitry controlling pluripotency and differentiation. Nature 2011, 477(7364):295-300. [10.1038/nature10398].

8. Ng SY, Johnson R, Stanton LW: Human long non-coding RNAs promote pluripotency and neuronal differentiation by association with chromatin modifiers and transcription factors. EMBO J 2012, 31(3):522-533. [10.1038/emboj.2011.459].

9. Rinn JL, Chang HY: Genome Regulation by Long Noncoding RNAs. Annu Rev Biochem 2012, 81:145-166.

10. Mitra SA, Mitra AP, Triche TJ: A Central Role for Long Non-coding RNA in Cancer. Frontiers in Genet 2012, 3(17).

11. Guttman $M$, Rinn JL: Modular regulatory principles of large non-coding RNAs. Nature 2012, 482(7385):339-346. [10.1038/nature10887].

12. Bernstein E, Allis CD: RNA meets chromatin. Genes \& Dev 2005, 19(14):1635-1655.

13. Cabili MN, Trapnell C, Goff L, Koziol M, Tazon-Vega B, Regev A, Rinn JL: Integrative annotation of human large intergenic noncoding RNAs reveals global properties and specific subclasses. Genes \& Dev 2011, 25(18):1915-1927.

14. Guttman M, Garber M, Levin JZ, Donaghey J, Robinson J, Adiconis X, Fan L, Koziol MJ, Gnirke A, Nusbaum C, Rinn JL, Lander ES, Regev A: Ab initio reconstruction of cell type-specific transcriptomes in mouse reveals the conserved multi-exonic structure of lincRNAs. Nat Biotech 2010, 28(5):503-510. [10.1038/nbt.1633].

15. Nagano T, Fraser $P$ : No-Nonsense Functions for Long Noncoding RNAs. Cell 2011, 145(2):178-181.

16. Pauli A, Valen E, Lin MF, Garber M, Vastenhouw NL, Levin JZ, Fan L, Sandelin A, Rinn JL, Regev A, Schier AF: Systematic identification of long noncoding RNAs expressed during zebrafish embryogenesis. Genome Res 2012, 22(3):577-591.

17. Mortazavi A, Williams BA, McCue K, Schaeffer L, Wold B: Mapping and quantifying mammalian transcriptomes by RNA-seq. Nat Methods 2008, 5:621-628. [10.1038/nmeth.1226].

18. Roberts A, Trapnell C, Donaghey J, Rinn J, Pachter L: Improving RNA-Seq expression estimates by correcting for fragment bias. Genome Biol 2011, 12(3):R22

19. Trapnell C, Pachter $L$, Salzberg SL: TopHat: discovering splice junctions with RNA-Seq. Bioinformatics 2009, 25(9):1105-1111.

20. Kozarewa I, Ning Z, Quail M, Sanders M, Berriman M, Turner D: Amplification-free Illumina sequencing-library preparation facilitates improved mapping and assembly of (G+C)-biased genomes. Nat Methods 2009, 6(4):291-295.

21. Trapnell C, Williams BA, Pertea G, Mortazavi A, Kwan G, van Baren MJ, Salzberg SL, Wold BJ, Pachter L: Transcript assembly and quantification by RNA-Seq reveals unannotated transcripts and isoform switching during cell differentiation. Nat Biotech 2010 28(5):511-515. [10.1038/nbt.1621].

22. Trapnell C, Roberts A, Goff L, Pertea G, Kim D, Kelley DR, Pimentel H, Salzberg SL, Rinn JL, Pachter L: Differential gene and transcript expression analysis of RNA-seq experiments with TopHat and Cufflinks. Nat Protoc 2012, 7(3):562-578. [10.1038/nprot.2012.016].

23. Garber M, Grabherr MG, Guttman M, Trapnell C: Computational methods for transcriptome annotation and quantification using RNA-seq. Nat Meth 2011, 8(6):469-477. [10.1038/nmeth.1613].

24. Nakaya H, Amaral P, Louro R, Lopes A, Fachel A, Moreira Y, El-Jundi T, da Silva A, Reis E, Verjovski-Almeida S: Genome mapping and expression analyses of human intronic noncoding RNAs reveal tissue-specific patterns and enrichment in genes related to regulation of transcription. Genome Biol 2007, 8(3):R43.

25. Okazaki Y, Furuno M, Kasukawa T, Adachi J, Bono H: Analysis of the mouse transcriptome based on functional annotation of 60,770 full-length cDNAs. Nature 2002, 420(6915):563-573. [10.1038/nature01266].

26. Dinger ME, Pang KC, Mercer TR, Mattick JS: Differentiating Protein-Coding and Noncoding RNA: Challenges and Ambiguities. PLoS Comput Biol 2008, 4(11):e1000176.

27. Tallack MR, Magor GW, Dartigues B, Sun L, Huang S, Fittock JM, Fry SV, Glazov EA, Bailey TL, Perkins AC: Novel roles for KLF1 in erythropoiesis revealed by mRNA-seq. Genome Research 2012.

28. Miller IJ, Bieker JJ: A novel, erythroid cell-specific murine transcription factor that binds to the CACCC element and is related to the Krüppel family of nuclear proteins. Mol Cell Biol 1993, 13(5):2776-2786.

29. Perkins AC, Sharpe AH, Orkin SH: Lethal [beta]-thalassaemia in mice lacking the erythroid CACCC-transcription factor EKLF. Nature 1995, 375(6529):318-322. [10.1038/375318a0]

30. Gene Expression Omnibus (GEO). [http://www.ncbi.nlm.nih.gov/geo/]

31. Langmead B, Trapnell C, Pop M, Salzberg S: Ultrafast and memory-efficient alignment of short DNA sequences to the human genome. Genome Bio/ 2009, 10(3):R25

32. Hubbard T, Barker D, Birney E, Cameron G, Chen Y, Clark L, Cox T, Cuff J, Curwen V, Down T, Durbin R, Eyras E, Gilbert J, Hammond M, Huminiecki L, Kasprzyk A, Lehvaslaiho H, Lijnzaad P, Melsopp C, Mongin E, Pettett R, Pocock M, Potter S, Rust A, Schmidt E, Searle S, Slater G, Smith J, Spooner W, Stabenau A, et al.: The Ensembl genome database project. Nucleic Acids Research 2002, 30:38-41

33. Pruitt KD, Tatusova T, Maglott DR: NCBI reference sequences (RefSeq): a curated non-redundant sequence database of genomes, transcripts and proteins. Nucleic Acids Res 2007, 35(suppl 1):D61-D65.

34. Illumina iGenomes. [http://cufflinks.cbcb.umd.edu/manual.html]

35. Hsu F, Kent WJ, Clawson H, Kuhn RM, Diekhans M, Haussler D: The UCSC Known Genes. Bioinformatics 2006, 22(9):1036-1046.

36. UCSC table browser. [http://genome.ucsc.edu/cgi-bin/hgTables? command=start]

37. Li H, Handsaker B, Wysoker A, Fennell T, Ruan J, Homer N, Marth G, Abecasis G, Durbin R, Subgroup GPDP: The Sequence Alignment/Map format and SAMtools. Bioinformatics 2009, 25(16):2078-2079.

38. Roberts A, Pimentel $H$, Trapnell $C$, Pachter L: Identification of nove transcripts in annotated genomes using RNA-Seq. Bioinformatics 2011, 27(17):2325-2329 
39. Robin X, Turck N, Hainard A, Tiberti N, Lisacek F, Sanchez JC, Müller M: pROC: an open-source package for $\mathrm{R}$ and $\mathrm{S}+$ to analyze and compare ROC curves. BMC Bioinformatics 2011, 12:77.

40. Lin MF, Jungreis I, Kellis M: PhyloCSF: a comparative genomics method to distinguish protein coding and non-coding regions. Bioinformatics 2011, 27(13):i275-i282.

41. Goecks J, Nekrutenko A, Taylor J, Team TG: Galaxy: a comprehensive approach for supporting accessible, reproducible, and transparent computational research in the life sciences. Genome Biology 2010, 11(8):R86.

42. Blankenberg D, Taylor J, Nekrutenko A, Team TG: Making whole genome multiple alignments usable for biologists. Bioinformatics 2011, 27(17):2426-2428.

43. Giardine B, Riemer C, Hardison RC, Burhans R, Elnitski L, Shah P, Zhang Y, Blankenberg D, Albert I, Taylor J, Miller W, Kent WJ, Nekrutenko A: Galaxy: A platform for interactive large-scale genome analysis. Genome Res 2005, 15(10):1451-1455.

44. Finn RD, Tate J, Mistry J, Coggill PC, Sammut SJ, Hotz HR, Ceric G, Forslund K, Eddy SR, Sonnhammer ELL, Bateman A: The Pfam protein families database. Nucleic Acids Res 2008, 36(suppl 1):D281-D288.

45. Benjamini $Y$ : Controlling the false discovery rate : a practical and powerful approach to multiple testing. J Royal Stat Soc 1995, 57:289-300.

46. Cufflinks manual. [http://cufflinks.cbcb.umd.edu/igenomes.html]

47. Wickham H: ggplot2: elegant graphics for data analysis. New York: Springer; 2009. [http://had.co.nz/ggplot2/book]

48. Bu D, Yu K, Sun S, Xie C, Skogerbø G, Miao R, Xiao H, Liao Q, Luo H, Zhao G, Zhao H, Liu Z, Liu C, Chen R, Zhao Y: NONCODE v3.0: integrative annotation of long noncoding RNAs. Nucleic Acids Res 2012 40(D1):D210-D215.

49. Livak KJ, Schmittgen TD: Analysis of Relative Gene Expression Data Using Real-Time Quantitative PCR and the $2^{-\Delta \Delta C_{T}}$ Method. 2001, 25(4):402-408.

50. Consortium TF, Carninci P, Kasukawa T, Katayama S, Gough J, Frith MC, Maeda N, Oyama R, Ravasi T, Lenhard B, Wells C, Kodzius R, Shimokawa K, Bajic VB, Brenner SE, Batalov S, Forrest ARR, Zavolan M, Davis MJ, Wilming LG, Aidinis V, Allen JE, Ambesi-Impiombato A, Apweiler R, Aturaliya RN, Bailey TL, Bansal M, Baxter L, Beisel KW, Bersano T, et al.: The

Transcriptional Landscape of the Mammalian Genome. Science 2005, 309(5740):1559-1563. [(Genome Network Project Core Group)].

51. Sam LT, Lipson D, Raz T, Cao X, Thompson J, Milos PM, Robinson D, Chinnaiyan AM, Kumar-Sinha C, Maher CA: A Comparison of Single Molecule and Amplification Based Sequencing of Cancer Transcriptomes. PLOS ONE 2011, 6(3):e17305.

doi:10.1186/1471-2105-13-331

Cite this article as: Sun et al:: Prediction of novel long non-coding RNAs based on RNA-Seq data of mouse Klf1 knockout study. BMC Bioinformatics 2012 13:331.

\section{Submit your next manuscript to BioMed Central} and take full advantage of:

- Convenient online submission

- Thorough peer review

- No space constraints or color figure charges

- Immediate publication on acceptance

- Inclusion in PubMed, CAS, Scopus and Google Scholar

- Research which is freely available for redistribution

Submit your manuscript at www.biomedcentral.com/submit
( Biomed Central 